\title{
Default Inferences in Metaphor Interpretation
}

\author{
Rodrigo Agerri, John Barnden, Mark Lee, and Alan Wallington \\ School of Computer Science, University of Birmingham \\ B15 2TT, Birmingham, UK \\ \{R.Agerri, J.A.Barnden, M.G. Lee, A.M.Wallington\}@C. . bham.ac.uk
}

\begin{abstract}
In this paper we provide a formalization of a set of default rules that we claim are required for the transfer of information such as causation, event rate and duration in the interpretation of metaphor. Such rules are domainindependent and are identified as invariant adjuncts to any conceptual metaphor. Furthermore, we show the role that these invariant mappings play in a semantic framework for metaphor interpretation.
\end{abstract}

\section{Introduction}

It is generally accepted that much of everyday language shows evidence of metaphor [1]. We assume the general view that metaphor understanding involves some notion of events, properties, relations, etc. that are transferred from a source domain into a target domain. In this view, a metaphorical utterance conveys information about the target domain. We are particularly interested in the metaphorical utterances that we call map-transcending. Consider the following example:

(1) "McEnroe starved Connors to death."

We do not address in this paper the issue of when an utterance is to be considered metaphorical. Instead, we aim to offer an explanation of how a metaphorical utterance such as (1) can be interpreted. If we infer, using our knowledge about McEnroe and Connors, that (1) is used to describe a tennis match, it can be understood as an example of the conceptual metaphors (or, in our terminology, 'metaphorical views') DEFEAT AS DEATH and NECESSITIES AS FOOD. However, these metaphorical views would not contain any relationship that maps the specific manner of dying that constitutes being starved to death (we say that "starving" is a map-transcending entity as it goes beyond known mappings). Yet one could argue that the manner of Connors's death is a crucial part of the informational contribution of (1).

A possible solution would be to create a new view-specific mapping that goes from the form of killing involved in starving to death to some process in sport, but such enrichment of mappings would be needed for many other verbs or verbal phrases that refer to other ways in which death is brought about, each requiring a specific specific mapping when occurring in a metaphorical utterance. Thus, finding adequate mappings could become an endless and computational intensive process. Moreover, there are even cases in which we may not find a plausible mapping. Consider the following description of the progress of a love affair:

B. Kokinov et al. (Eds.): CONTEXT 2007, LNAI 4635, pp. 1-14 2007.

(C) Springer-Verlag Berlin Heidelberg 2007 
(2) "We're spinning our wheels."

It is not very clear what could be a target correspondent for 'wheels'; the unavailability of a correspondent would therefore prevent the source to target transfer of information needed for the interpretation of the metaphorical utterance. Thus, an account of metaphor ought to explain what extra information map-transcending entities provide. Furthermore, how the transfer of information occurs should be account for in a viable computational manner.

ATT-Meta [2] is an AI System and approach to metaphor interpretation that, apart from providing functionalities such as uncertainty and conflict handling [3], introduces two features central to the interpretation of metaphorical utterances such as (1) and (2): Instead of attempting the creation of new mappings to extend an existing metaphorical view, ATT-Meta employs query-driven reasoning within the terms of the source domain using various sources of information including world and linguistic knowledge. The reasoning connects unmapped ideas used by utterances, such as wheels and starving, to other source-domain ideas for which a mapping is already known. These known mappings may be constituents of a particular metaphorical view, but previous work [4 5] has shown evidence that there metaphorical aspects (such as relations and properties between events) that, subject to being called, invariantly map from source to target (we call these mappings View-Neutral Mapping Adjuncts or VNMAs). These allow many mapping effects, which would otherwise have to be duplicated across all view-specific mappings, to be factored out into separate mappings.

In our approach, source domain reasoning takes place in a special, protected computational context that we call the "pretence context". We use the term 'reality' to refer to the space outside the pretence where propositions are about reality as the understander sees it. The nature of source domain reasoning in metaphor interpretation has not previously been adequately investigated, although a few authors have addressed it to a limited extent [6789].

Currently ATT-Meta implements the VNMAs by including them in view-specific rules, but we plan to make the system more modular and its view-specific mappings more economical by implementing VNMAs as separate default rules. The first step towards that goal is to provide a formalization of these mappings and to clarify their role in metaphor interpretation. In order to do so, we embed them in a semantic framework for metaphor interpretation inspired by Segmented Discourse Representation Theory [10] tailored to capture the main aspects of the ATT-Meta approach. In this sense, it is not an aim of this paper to propose an SDRT-based account of metaphor but instead adapt its semantic representation structures to represent the ATT-Meta view on metaphor understanding. Other authors seem to have merely assumed the existence of a special type of invariant mappings similar to the VNMAs [1] but they do not address the issue explicitly, aside from the early work of Carbonell [6].

The next section briefly describes the ATT-Meta approach focusing on source domain inferencing and VNMAs. Section 3 describes a number of VNMAs that are used to interpret various metaphorical utterances. In section 4 we discuss the main components to be included in a semantic account of metaphor. We then propose to adapt the SDRT formal framework to our purposes of providing a semantic account of metaphor interpretation based on the ATT-Meta approach. Section 5 shows how VNMAs can ex- 
plain examples discussed by other authors in a uniform and systematic manner. Section 6 presents some conclusions and discussion on further work.

\section{Within-Pretence Inference and Invariant Mappings}

Let us go back to example (1):

(1) "McEnroe starved Connors to death."

Assuming a commonsensical view of the world and if (1) is being used metaphorically to describe the result of a tennis match, a plausible target interpretation would be that McEnroe defeated Connors by performing some actions to deprive him of his usual playing style. In the ATT-Meta approach, within-pretence inferencing produces a proposition to which we may apply a mapping to transfer that information. An important feature of the pretence space is that it takes the meaning of source domain utterances as literal. Thus, and assuming a commonsensical view of the world, a within-pretence meaning would be that McEnroe starved Connors to death in a biological sense. The inferencing within the pretence can then conclude that McEnroe caused Connors's death by depriving or disabling him. Leaving some details aside, the partial logical form (in the pretence) of the metaphorical utterance (1) may be represented as follows (without taking into account temporal issues):

(i) $\exists x, y, e(\operatorname{McEnroe}(x) \wedge$ Connors $(y) \wedge \operatorname{starve}-$ to - death $(e, x, y))$

This says that there is an event $e$ of $x$ starving $y$ to death (we use the notion of event á la Hobbs [11] to describe situations, processes, states, etc.). It may be suggested that if we were trying to map the partial expression (i), its correspondent proposition in the target could be expressed by this formula:

(ii) $\exists x, y, e(\operatorname{McEnroe}(x) \wedge \operatorname{Connors}(y) \wedge \operatorname{defeat}(e, x, y))$

According to this, the event of $x$ defeating $y$ in the reality would correspond to the event of $x$ starving $y$ to death in the pretence. However, by saying "McEnroe starved Connors to death" instead of simply "McEnroe killed Connors" the speaker is not merely intending to convey that McEnroe defeated Connors, but rather something related to the manner in which Connors was defeated. Following this, starving may be decomposed into the cause $e_{1}$ and its effect, namely, "being deprived of food":

(iii) $\exists x, y, z, e_{1}, e_{2}, e_{3}\left(\operatorname{McEnroe}(x) \wedge \operatorname{Connors}(y) \wedge \operatorname{food}(z) \wedge \operatorname{starve}\left(e_{1}, x, y\right) \wedge\right.$ $\left.\operatorname{death}\left(e_{2}, y\right) \wedge \operatorname{deprived}\left(e_{3}, y, z\right) \wedge \operatorname{cause}\left(e_{1}, e_{3}\right)\right)$

Note that by factoring out "starving to death" in this way we not only distinguish the cause from the effect but doing so allows us to establish a relation between "death" in the pretence to "defeat" in reality using the known mapping in DEFEAT AS DEATH (and possibly "starving" to "McEnroe's playing" although we will not press this issue here).

Now, by means of lexical information regarding "starving", it can be inferred that McEnroe deprived Connors of a necessity (see, e.g., Wordnet), namely, of the food 
required for his normal functioning (the NECESSITIES AS FOOD metaphorical view would provide mappings to transfer food to the type of shots that Connors needs to play his normal game). In other words, Connors is defeated by the particular means of depriving him of a necessity (food) which means that being deprived causes Connors's defeat. This fits well with the interpretation of (1) where McEnroe's playing deprived Connors of his usual game. Moreover, linguistic knowledge also provides the fact that starving someone to death is a gradual, slow process. The result of within-pretence inferencing may be represented as follows:

(iv) $\exists x, y, z, e_{1}, e_{2}, e_{3}\left(\operatorname{McEnroe}(x) \wedge \operatorname{Connors}(y) \wedge \operatorname{food}(z) \wedge \operatorname{starve}\left(e_{1}, x, y\right) \wedge\right.$ $\operatorname{death}\left(e_{2}, y\right) \wedge$ deprived $\left.\left(e_{3}, y, z\right) \wedge \operatorname{cause}\left(e_{1}, e_{3}\right) \wedge \operatorname{cause}\left(e_{3}, e_{2}\right) \wedge \operatorname{rate}\left(e_{1}, \operatorname{slow}\right)\right)$

'Slow' refers to a commonsensical concept in the pretence related to the progress rate of starving. Now, the existing mapping DEFEAT AS DEATH can be applied to derive, outside the pretence, that McEnroe defeated Connors, but no correspondences are available to account for the fact that McEnroe caused the defeat of Connors by depriving him of his normal play. Furthermore, the same problem arises when trying to map the slow progress rate of a process like starving.

In the ATT-Meta approach to metaphor interpretation, the mappings of caused and rate discussed above are accomplished by a type of default mappings that we specify as VNMAs (the Causation and Rate VNMAs, respectively; see [12] for an informal but detailed description of a number of VNMAs). VNMAs account for the mapping of aspects of the source domain that do not belong to a specific metaphorical view but that often carry an important informational contribution (or even the main one) of the metaphorical utterance. These source domain aspects can be captured as relationships and properties (causation, rate, etc.) between two events or entities that, subject to being called, identically transfer from the pretence to the reality.

Summarizing, the following processes, amongst others, are involved in the understanding of map-transcending utterances: 1) Construction of within-pretence domain meaning of the utterance. 2) Placing of it in the pretence context. 3) Source-domain reasoning within the pretence cocoon, using the direct meaning constructed in 1) with world and linguistic knowledge about the source domain. 4) Transfers by application of specific mappings in metaphorical views and often invariant mappings specified as VNMAs. The remaining of the paper focuses on the characterization and formalization of VNMAs from a semantic point of view.

\section{Description of VNMAs}

By using VNMAs and within-pretence inferencing, we do not need to extend the mappings in the metaphorical view to include information about "depriving of a necessity", "food" or "causing Connors's death". VNMAs transfer those properties or relations between mappees that are view-neutral. Moreover, VNMAs are parasitic on the metaphorical views in the sense that they depend on some mappings to be established for the VNMAs to be triggered. That is why VNMAs are merely "adjuncts". VNMAs can also be seen as pragmatic principles that guide the understanding of metaphor by transferring aspects of the source domain that remain invariant. 
In example (1), there are two VNMAs involved in the transfer of the causation and the "slowness", namely, the Causation and Rate VNMAs which are described below. Additionally, we also discuss two more VNMAs related to the temporal order of events and the value-judgement that agents assign to events (others are described in [4[5]12]).

\subsection{Causation/Ability}

The idea is that there are relationships and properties (causation, (dis)enablement, etc.) between two events or entities that identically transfer from the pretence to the reality. We use the $\mapsto$ symbol to express that this mapping is a default.

Causation/Ability VNMA: "Causation, prevention, helping, ability, (dis)enablement and easiness/difficulty relationships or properties of events between events or other entities in the pretence, map to those relationships between their mappees (if they have any) in the reality." The invariant mapping involved in the interpretation of (1) could be represented as follows:

Causation: $\forall e_{1}, e_{2}\left(\operatorname{cause}\left(e_{1}, e_{2}\right)_{\text {pret }} \mapsto \operatorname{cause}\left(e_{1}, e_{2}\right)_{r l t}\right)$

As an additional note, the specific mapping of each event or state variable does not depend on the VNMA but on the metaphorical view in play. For example, if we consider the contemporary situation in which McEnroe and Connors are tennis pundits on TV, we may need a metaphorical view such as ARGUMENT AS WAR to interpret the utterance "McEnroe starved Connors to death". In other words, VNMAs do not themselves establish the mappees between the pretence and the reality.

\subsection{Rate}

Rate: "Qualitative rate of progress of an event in the source domain maps identically to qualitative rate of progress of its mappee. E.g., if an event progresses slowly (in the context of the everyday commonsensical world), then its mappee progresses slowly (in the target context)".

Consider the following utterance:

(3) My car gulps gasoline.

Briefly, the metaphorical view involved is MACHINES AS CREATURES, that maps biological activity to mechanical activity. The within-pretence reasoning may be performed along the following lines: It can be inferred in the pretence that gasoline helps the car to be alive, therefore, it helps the car to be biologically active. The Causation/Ability VNMA (which deals with helping) combined with the above metaphorical view provide the target domain contribution that gasoline helps the car to run. Given that we can assume that an act of gulping is normally moderately fast the use of the Rate VNMA allows us to conclude that the car's use of gasoline is moderately fast. The logical form of this VNMA is could be expressed as follows:

Rate: $\forall e, r\left(\right.$ rate $\left.(e, r)_{\text {pret }} \mapsto \operatorname{rate}(e, r)_{r l t}\right)$

If the rate an event $e$ in the pretence is $r$, then the rate maps to the mappee event in the reality, that is, it also has rate $r ; r$ refers to the qualitative rate of progress or duration of an specific event $e$. 


\subsection{Time-Order}

Time-Order: "The time order of events in a source domain is the same as that of their mappee events, if any".

Time-order is quite useful for map-transcending examples such as

(4) McEnroe stopped hustling Connors.

We might infer in the pretence that McEnroe was once hustling Connors which would be transferred by the Time-Order VNMA. For the formalization of this VNMA, we say that if event $e_{1}$ precedes event $e_{2}$ in the pretence, then the mappee events in the reality exhibit the same ordering.

Time-Order: $\forall e_{1}, e_{2}\left(\right.$ precede $\left.\left(e_{1}, e_{2}\right)_{\text {pret }} \mapsto \operatorname{precede}\left(e_{1}, e_{2}\right)_{r l t}\right)$

\subsection{Value-Judgement}

Value-Judgement: "Level of goodness, importance or other types of value assigned by the understander to states of affairs in the source domain map identically to levels of goodness, etc., of their mappee states of affairs, if any."

(5) That is a gem of an idea.

We could argue that a metaphorical view IDEAS AS OBJECTS is used to interpret this example. However, this view does not provide correspondences for mapping the fact that we see 'gems' as valuable or precious. Instead of trying to find correspondents to map different types of objects to different types of ideas we assume that it is possible to infer in the pretence that gems are valuable and this value judgement about objects that are gems is transfer by the Value-Judgement VNMA. This mapping is expressed by the following formula:

Value-Judgement: $\forall e, v\left(v a l u e(e, v)_{\text {pret }} \mapsto \operatorname{value}(e, v)_{r l t}\right)$

\section{A Semantic Framework for Metaphor Interpretation}

Embedding the VNMAs in a semantic framework for metaphor interpretation is useful as a first step towards their implementation as default rules in the ATT-Meta system, but it is also interesting in its own right to show the contribution that the ATT-Meta approach can make towards a semantics of metaphor.

\subsection{A Contextual and Query-Driven Approach}

In the somewhat simplified discussion on the within-pretence reasoning and mappings necessary to interpret metaphorical utterances such as (1), we have not paid too much attention two main issues. Firstly, the actual source domain reasoning performed by the ATT-Meta system is query-driven. 
Although in previous sections we used various sources of contextual information to license certain within-pretence inferences, we have only considered isolated metaphorical utterances, and metaphor understanding has been pictured as a process of forward reasoning from the direct meaning of utterances (in the pretence) and then the application of various metaphorical mappings to the result of source domain reasoning to arrive at the informational contributions in the target. Moreover, other possible inferences that could be drawn were ignored without specifying any principles or criteria whereby the reasoning could be guided towards the particular informational contributions discussed. The notion of discourse-query-directed reasoning provides such guidance. When analyzing previous examples, we assume that the surrounding discourse context supplies queries that guide source domain reasoning in broadly the reverse order to that in which we described them in section 2 (see [13] for a detailed description of query-directed reasoning in ATT-Meta). Other authors such as Hobbs [7] and Asher and Lascarides [14] also acknowledge the importance of context-derived reasoning queries in the interpretation of metaphorical utterances.

We are not claiming that query-directed reasoning may be the only type of reasoning involved in the processing of metaphor, but it seems to be particularly important in the processing of connected discourse. Although the ATT-Meta system at present works with single-sentence utterances (albeit with the aid of discourse-query-directed reasoning), an aim for future versions is to extend it to the processing of discourse, and the semantic framework will need to allow for this.

Secondly, we have been using various sources of contextual knowledge that interact in the processing of the utterance: a) View-specific mappings provided by the relevant metaphorical views (DEFEAT AS DEATH and NECESSITIES AS FOOD); b) Linguistic and contextual information necessary for reasoning in the pretence; c) Relations and properties between events such as causation and rate that are inferred in the pretence; d) VNMAs that transfer within-pretence event relations and properties to reality. In our view, a suitable semantic approach to metaphor should include at least these five components.

\subsection{VNMAs in a Semantic Framework}

Metaphor is a highly contextual phenomenon, and one of the most interesting semantic approaches that model context are dynamic semantics such as Discourse Representation Theory [15] which views meaning as a relation between the contexts that compose a discourse. Furthermore, we are interested in representing relations between events such as causation and temporal order in the pretence context.

There are two prominent computationally-oriented semantic approaches [16]10] that take into account contextual and linguistic information to stress the importance of relations between semantic representations of events in discourse interpretation. Specifically, we adapt the semantic representation procedure of Segmented Discourse Representation Theory or SDRT [10] to build pretence contexts as Segmented Discourse Representation Structures (SDRSs) consisting of the result of source domain reasoning. The conclusion of within-pretence inference can in turn be mapped to reality by using various view-specific mappings and VNMAs. In other words, we can see the pretence SDRS as the input for what the ATT-Meta system does when interpreting 
metaphor - it will reason with it, producing an output of inferred reality facts which we may also represent by means of an SDRS. The result of reasoning in the pretence context to interpret (1) would now looks as follows:

PRET:

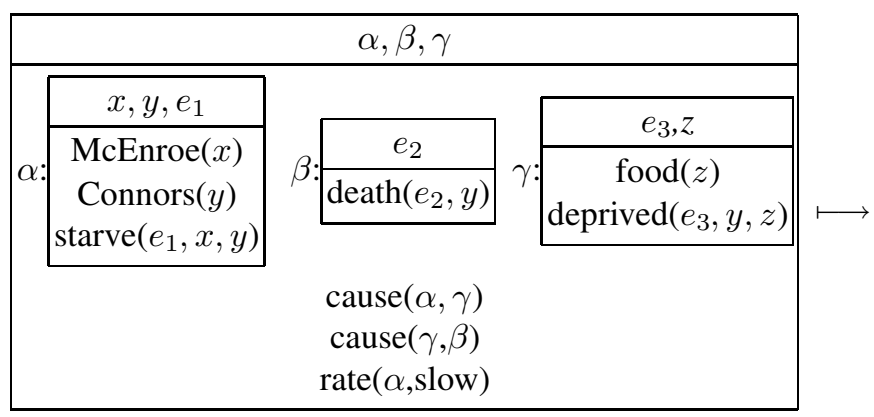

where $\alpha$ and $\beta$ are labels for DRSs representing events, PRET for a pretence space and $\longmapsto$ mappings (VNMAs and central mappings) needed in the interpretation of the metaphorical utterance. Importantly, the VNMAs would pick upon aspects such as causation and rate from pretence to transfer them to reality producing an output which could also be represented as a SDRS:

RLT:

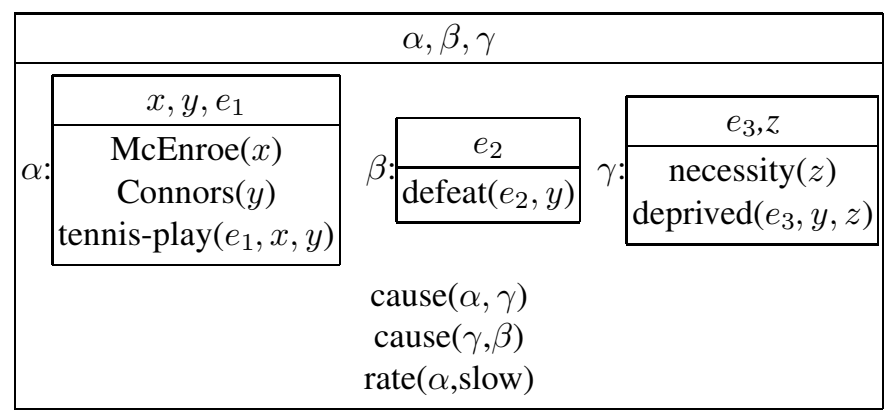

Note that this formal representation integrates the systematicity of mapping invariantly certain aspects of metaphorical utterances by formulating them as relations and properties of events that can be represented as relations and properties of DRSs. For this purpose we will need to modify the construction rules of SDRSs to be able to infer properties and relations involving individuals $(x, y, \ldots)$ and not only DRSs' labels such as $\alpha$ and $\beta$. In addition to this, we need to capture the interaction of the various sources of information used (linguistic knowledge, world knowledge, etc.) to infer causation and rate in the pretence. Thus, we partially adopt SDRT formal framework to represent ATT-Meta's within-pretence reasoning, event relations, event properties and VNMAs with the purpose of developing a semantic account of metaphor interpretation.

\subsection{Context and Knowledge}

Within-pretence reasoning partially relies on inferences provided by the discourse context and linguistic and world knowledge. In the ATT-Meta system, world knowledge 
roughly corresponds to source domain knowledge. On the one hand, we have been using our commonsensical knowledge about McEnroe and Connors to interpret example (1) as metaphorically describing a tennis match. On the other hand, linguistic knowledge is used to pretend that the direct meaning of the metaphorical utterance is true, which allows us to derive causation and rate. Thus, we assume that the understander possesses some world knowledge that provides information about "starving someone to death":

- If $e_{3}$ where $y$ is deprived and $e_{1}$ where $x$ starves $y$ are connected, then by default, $e_{1}$ causes $e_{3}$.

- If $e_{2}$ where $y$ dies and $e_{3}$ where $y$ is deprived are connected, then by default, $e_{3}$ causes $e_{2}$.

- If $e_{1}$ where $x$ starves $y$, then by default, the rate of progress of $e_{1}$ is slow.

Furthermore, common sense about causation tells us that "if $e_{1}$ causes $e_{3}$ then $e_{3}$ does not occur before $e_{1}$ ". Following this, the knowledge needed to interpret example (3) needs to include the that the drinking rate is fast:

If $e$ where $x$ gulps, then by default, $x$ in $e$ drinks moderately fast.

Asher and Lascarides use a non-monotonic logic (Commonsense Entailment) which is designed to handle reasoning with conflicting knowledge resources. SDRT specifies where in the preceding discourse structure the proposition introduced by the current sentence can attach with a discourse relation. In order to do that, it is necessary to provide a set of rules for the understander to infer which discourse relation should be used to do attachment. We adopt a similar notation to represent discourse update (see [10] for details on the discourse update function) so that defeasible knowledge about causation, rate, temporal order, etc., allows the inference of event relations and properties in the pretence.

Let us suppose that in a context (pretence) pret 1 we want to attach some event denoted by $\beta$ to $\alpha$, such that $\langle$ pret, $\alpha, \beta\rangle$. This update function can be read as "the representation pret of a text so far is to be updated with the representation $\beta$ of an event via a discourse relation with $\alpha$ " [10]. Let $\sim$ represent a defeasible connective as a conditional, and let $e v(\alpha)$ stand for "the event described in $\alpha$ "; although $e v(\alpha)$ is quite similar to the notion of main eventuality me defined by Asher and Lascarides [10], we do not commit to other assumptions of their theory.

Thus, some of the source domain knowledge about causation in (1) discussed above could now be represented as follows:

$$
\begin{aligned}
& \langle\text { pret }, \alpha, \beta\rangle \text { dies }(\text { connors, ev }(\beta)) \wedge \text { starves }(\text { mcenroe, connors, ev }(\alpha)) \\
& \sim \operatorname{cause}(\operatorname{ev}(\alpha), \operatorname{ev}(\beta))
\end{aligned}
$$

We can then infer in the pretence a causation relation between $\alpha$ and $\beta$ if the event represented in $\alpha$ normally causes $\beta$ :

Causation: $\langle$ pret $, \alpha, \beta\rangle \wedge(\operatorname{cause}(e v(\alpha), e v(\beta)) \leadsto \operatorname{causation}(\alpha, \beta)$

${ }^{1}$ See Lee and Barnden [17] for cases in which more than one pretence is involved in the interpretation of metaphor. 
Note that 'cause' refers to the epistemic notion of one event causing another, whereas 'causation' refers to an inferred semantic relation between segments of discourse or, in other words, between semantic representation of events by means of DRSs. In order to include properties (and not only relations) in this framework, we assume a conceptualist point of view and consider that properties such as rate or value-judgement denote concepts (fast, slow, good, bad) which may correspond to the absolute rate in a commonsensical view of the world. Its representation in our semantic framework could be defined by adding an extra clause to the definition of DRS-formulae:

- If $P$ is a property symbol and $\alpha$ and $r$ are an episode label and a property label respectively, then $P(\alpha, r)$ is an DRS-formula (see [10] for the complete definitions of DRS-formulae and SDRS construction).

Thus, a rule encoding contextual knowledge to infer rate in the pretence would look as follows (note that when considering event properties we only need to consider one DRS $\alpha$ in our rules, even though a discourse usually consists of one or more DRSs):

$$
\langle\text { pret, } \alpha\rangle \text { gulps(car, gasoline, ev }(\alpha)) \leadsto \operatorname{fast}(\operatorname{ev}(\alpha))
$$

Supported by this rule we can then infer an event property in the pretence for its subsequent transfer to reality via the Rate VNMA (when the Rate VNMA is instantiated):

Rate: $\langle$ pret,$\alpha\rangle(\operatorname{fast}(e v(\alpha)) \leadsto \operatorname{rate}(\alpha$, fast $)$

\subsection{VNMAs Revisited}

Section 3 described several VNMAs and showed their contribution to the analysis of four different metaphors. VNMAs are considered to be default mapping rules that transfer relations and properties from pretence contexts to reality. Furthermore, we claim that VNMAs are adjunct to central mappings provided by the metaphorical view(s) (DEFEAT AS DEATH, IDEAS AS OBJECTS) used in the utterance context.

We use the VNMAs introduced in section 3 and the above points about withinpretence inferencing and contextual knowledge to offer SDRT-based semantic representations for an analysis of examples (3) and (4) based on the ATT-Meta approach to metaphor. We leave out any details not directly relevant to the discussion on VNMAs.

We claimed in section 3 that the transfer to reality of the within-pretence information relative to how fast the car drinks/uses gasoline (derived from linguistic knowledge about "gulp") was performed via a Rate VNMA. The following (partial) picture of a discourse captures this:

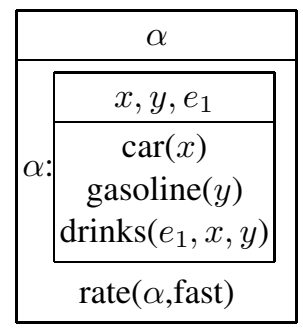


'Fast' refers to a commonsensical concept in the pretence related to the rate of "gulping". From here, the Rate VNMA would transfer rate $(\alpha, f a s t)$ to reality. We do not represent the correspondent representation for the sake of brevity. Note that we are only considering the aspects directly involved in the use of Rate VNMA, and as such we do not include the discourse in which an utterance such as (3) may occur. It should be stressed that the context-driven character of the ATT-Meta approach accounts for the transfer of a feature such as rate in a discourse context in which the consumption of gasoline is being considered. Thus, features other than rate could be important in other uses of 'gulp' (e.g., such as the fact that gulping usually refers to a noisy way of drinking).

We follow the same process with respect to example (4) involving time-order:

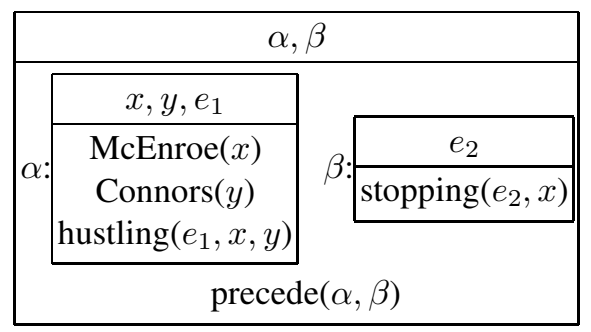

Summarizing, the semantic framework outlined in this section consists of:

- DRSs and SDRSs consisting of events, individuals, states, etc. They can be thought of as situations or as representation structures as in dynamic semantics. A context consists of one or more DRSs, DRSs relations and properties.

- Event relations and properties such as causation, rate, time-order, value-judgement, etc inferred in the pretence for the systematic transfer of certain type of information conveyed by metaphorical utterances. The transfer of this type of information via VNMAs is a contribution of the ATT-Meta approach to metaphor interpretation [4]5].

\section{Comparison to Other Approaches}

Following the ATT-Meta claim that often convey crucial information via VNMAs, some examples of metaphor proposed by other authors. Consider the following utterance:

(6) Sam is a pebble.

Asher and Lascarides [14] claim that it is not possible to calculate the meaning of an utterance such as (6) on the basis of the domain information about pebbles, but that it is possible to process it if it is discourse related to other utterance, e.g., as in "John is a rock but Sam is a pebble" (we could infer a Contrast rhetorical relation, which would help us to work out the metaphorical meaning of (6)). However, by using VNMAs and within-pretence reasoning an addressee of (6) may reach an interpretation without necessarily needing a Contrast rhetorical relation to guide the reasoning. In our 
case, linguistic knowledge and within-pretence reasoning about 'pebbles' establish that they are small, and a very frequent association of unimportant entities with "small size" allows the defeasible inference that something is low, inferior, limited in worth (see Wordnet or any other lexical database). Using the Value-Judgment VNMA ("Levels of goodness, importance, etc., assigned by the understander in the source domain map identically to levels of goodness, etc."), we can convey the meaning that Sam is limited in worth (worthless). Like Asher and Lascarides, Hobbs also claims that inferring discourse structure is crucial to understand certain metaphorical utterances [16]:

(7) John is an elephant.

Which Hobbs argues can only be interpreted if we add extra information so that it now reads:

(8) Mary is graceful but John is an elephant.

Which now allows us to infer Contrast in order to work out the meaning of "John being an elephant" as oppose to "Mary being graceful". A VNMA approach is not so reliant on rhetorical relations such as Contrast, which, in our view, could be an advantage to analyze certain examples. In fact, it seems that there are cases in which inferring a discourse relation is not enough to interpret the metaphorical utterance. Consider the following utterance:

(9) Mary is a fox and John is an elephant.

In this case, we can infer a Coordination relation to account for the conjunction of the two segments [18]. However, it seems that inferring Coordination would not be enough to guide the interpretation of (9) towards some attributes of Mary (e.g., being cunning, small, etc.) and John (big, having good memory, etc.). In our approach, and subject to the appropriate contextual query to be provided by the discourse, size-related features might be transferred by a Physical Size VNMA; in an appropriate context (9) could also be used to convey that John has a good memory and that Mary is cunning. In this case, forgetfulness could be seen a tendency to perform a mental act of a certain type and non-forgetfulness could be handled by a Negation VNMA, Mental states VNMA and a Event-Shape VNMA (for tendencies).

Studying the interaction, if any, between VNMAs and discourse relations may allow us to naturally extend the study of metaphor to discourse. However, this issue exceeds the purposes of this paper. In any case, it seems that information relative to events rate, duration, value-judgement, etc., cannot be captured solely by means of rhetorical relations.

With respect to the context-driven character of the ATT-Meta approach, a further issue could be raised, namely, why consider principles such as VNMAs at all? It could just be that the types of features transferred by VNMAs happen to be commonly transferred things, and any feature could be transferred in a view-neutral way, if context asks for it. However, there is in fact evidence that not all types of features can be viewneutrally transferred. Suppose that there are two long, thin pencils, on red and the other yellow. Following the contrast-based examples discussed above, it would be very odd if someone utters 
(10) Peter's pencil is yellow, but Mike's is a tomato.

The use of 'tomato' to describe a pencil only would seem to appropriate if the pencil were quite short and bulbous, which would mean that it will very difficult to transfer the colour without also mapping the shape. We face similar problems when trying to analyze utterances such as

(11) Peter has dark hair but Mike is an elephant.

just because the latter had grey hair. In this sense, we are a taking a conservative approach, only proposing that a particular type of feature is view-neutrally transferred if we have evidence.

\section{Concluding Remarks}

This paper investigates the formalization and semantic representation of the ATT-Meta approach to metaphor interpretation. The ATT-Meta approach is backed up by a powerful implementation that performs sophisticated reasoning to interpret metaphorical utterances. We have focused on description and formalization of several VNMAs, mappings for the systematic transference of invariant aspects from source to target. We have shown how a dynamic semantic approach can be adapted for these purposes to offer an unified semantic representation of ATT-Meta's view of metaphor interpretation.

Map-transcending entities pose a problem for several analogy-based approaches to metaphor interpretation, both from a computational and a theoretical point of view. With respect to the computational approaches, theories of metaphor interpretation based on analogy [1920] usually require a conceptual similarity between the source and the target domains. Map-transcending entities need to be mapped by extending on the fly the metaphorical views with new correspondences. We have argued that this strategy is both computationally expensive and in some cases, plainly impossible.

Formal semantic approaches [10] do not account for metaphorical utterances including map-transcending entities. Other works [67|8|9] have addressed source domain reasoning to a limited extent, but its role in metaphor interpretation has not previously been adequately investigated. Moreover, map-transcending entities pose a problem for analogy-based approaches to metaphor interpretation [19], which usually require a conceptual similarity between the source and the target domains.

\section{References}

1. Lakoff, G., Johnson, M.: Metaphors We Live By. University of Chicago Press, Chicago (1980)

2. Barnden, J., Lee, M.: An artificial intelligence approach to metaphor understanding. Theoria et Historia Scientarum 6, 399-412 (2002)

3. Barnden, J.: Uncertainty and conflict handling in the att-meta context-based system for metaphorical reasoning. In: Akman, V., Bouquet, P., Thomason, R.H., Young, R.A. (eds.) CONTEXT 2001. LNCS (LNAI), vol. 2116, pp. 15-29. Springer, Heidelberg (2001) 
4. Barnden, J., Glasbey, S., Lee, M., Wallington, A.: Domain-transcending mappings in a system for metaphorical reasoning. In: Conference Companion to the 10th Conference of the European Chapter of the Association for Computational Linguistics (EACL 2003), pp. 57$61(2003)$

5. Wallington, A., Barnden, J., Glasbey, S., Lee, M.: Metaphorical reasoning with an economical set of mappings. Delta 22 (2006)

6. Carbonell, J.: Metaphor: An inescapable phenomenon in natural-language comprehension. In: Lehnert, W., Ringle, M. (eds.) Strategies for Natural Language Processing, pp. 415-434. Lawrence Erlbaum, Hillsdale (1982)

7. Hobbs, J.: Literature and Cognition. CSLI, Lecture Notes, Stanford (1990)

8. Martin, J.: A computational model of metaphor interpretation. Academic Press, New York (1990)

9. Narayanan, S.: KARMA: Knowledge-based action representations for metaphor and aspect. $\mathrm{PhD}$ thesis, Computer Science Division, EECS Department, University of California, Berkeley (1997)

10. Asher, N., Lascarides, A.: Logics of Conversation. Cambridge University Press, Cambridge (2003)

11. Hobbs, J.: Ontological promiscuity. In: Annual Meeting of the ACL, Chicago, pp. 61-69 (1985)

12. Wallington, A., Barnden, J.: Similarity as a basis for metaphor: Invariant transfer and the role of VNMAs. Technical Report CSRP-06-02, School of Computer Science, Univ. of Birmingham (2006)

13. Barnden, J., Lee, M.: Understanding open-ended usages of familiar conceptual metaphors: An approach and artificial intelligence system. Technical report, Technical Report CSRP-0105, School of Computer Science, Univ. of Birmingham (2001)

14. Asher, N., Lascarides, A.: The semantics and pragmatics of metaphor. In: Bouillon, P., Busa, F. (eds.) The Language of Word Meaning, pp. 262-289. Cambridge University Press, Cambridge (2001)

15. Kamp, H., Reyle, U.: From Discourse to Logic: Introduction to Modeltheoretic semantics of natural language, formal language and Discourse Representation Theory. Kluwer Academic Publishers, Dordrecht (1993)

16. Hobbs, J.: An approach to the structure of discourse. In: Everett, D. (ed.) Discourse: Linguistic, Computational and Philosophical Perspectives (1996)

17. Lee, M., Barnden, J.: Reasoning about mixed metaphors with an implemented ai system. Metaphor and Symbol 16, 29-42 (2001)

18. Gomez-Txurruka, I.: The natural language conjunction 'and'. Linguistics and Philosophy 26, 255-285 (2003)

19. Falkenhainer, B., Forbus, K., Gentner, D.: The structure-mapping engine: algorithm and examples. Artificial Intelligence 41, 1-63 (1989)

20. Holyoak, K., Thagard, P.: Analogical mapping by constraint satisfaction. Cognitive Science 13, 295-355 (1989) 